\title{
Low Dose Electron Microscopy of Amonium Urates
}

Hector Calderon ${ }^{1}$, Jeffrey Rimer ${ }^{2}$, Francisco Robles-Hernandez ${ }^{2}$ and Christian Kisielowski ${ }^{3}$

${ }^{1}$ Instituto Politecnico Nacion ESFM, Mexico, Distrito Federal, Mexico, ${ }^{2}$ University of Houston, Houston, Texas, United States, ${ }^{3}$ The Molecular Foundry and Joint center for Artifical Photosynthesis, Berkeley, California, United States

Uric acid is a heterocylic organic compound generated in the liver, intestines and kidneys. Uricacid is insoluble in organics, but it dissolves in water at room temperature. A large number of health concerns are associated to uric acid precipitation i.e., crystals. Such crystals are the main component of human kidneys stones (calculi). Recently, the study of uric acid has generated a great deal of interest mainly in an effort to identify its nature in order to develop potential techniques to prevent their crystallization and growth $\mathrm{n}$ the human body. As of today, the scientific community has used different techniques, from wetchemistry and conventional characterization to advanced electron microscopy. Here a transmission electron microscopy work is presented where the sensitivity of the material together with the needs for a complete electron microscopy characterization are given, additionally there is an analysis of images by means of specialized image analysis software. The main technique in use is TEM but in a rather low dose mode and in an aberration corrected electron microscope so as to achieve atomic resolution and a good spatial resolution. The result is that the molecular arrangement of urates can be clearly determined from the achieved images.

The TEAM 1.0 electron microscope from NCE-MF at the LBNL is used at $80 \mathrm{KeV}$ and in low dose below $10 \mathrm{e}^{-} / \mathrm{A}^{2} \mathrm{~s}$. Images series are used taken at a definite focus. The stability of the sample can be preserved only for such a low number of images taken for $1 \mathrm{~s}$. A relatively good contrast together with atomic resolution is obtained after overlapping the aligned images. In all cases the software MacTempass $\mathrm{X}$ is used. The images taken after $10 \mathrm{sec}$ start showing the effect of beam sample interaction very clearly thus the focal series and overlapping must be limited in number. Nevertheless, the molecular structure of the Urate crystals can be easily identified in the final produced images. Urate crystals can be straight or curved. Interestingly, curved urates develop dislocations, missing atomic planes can be identified in the atomic resolution images of curved urates with distance separation proportional to the crystal curvature. The analogy with inorganic crystal is surprisingly high in this respect [1]. 


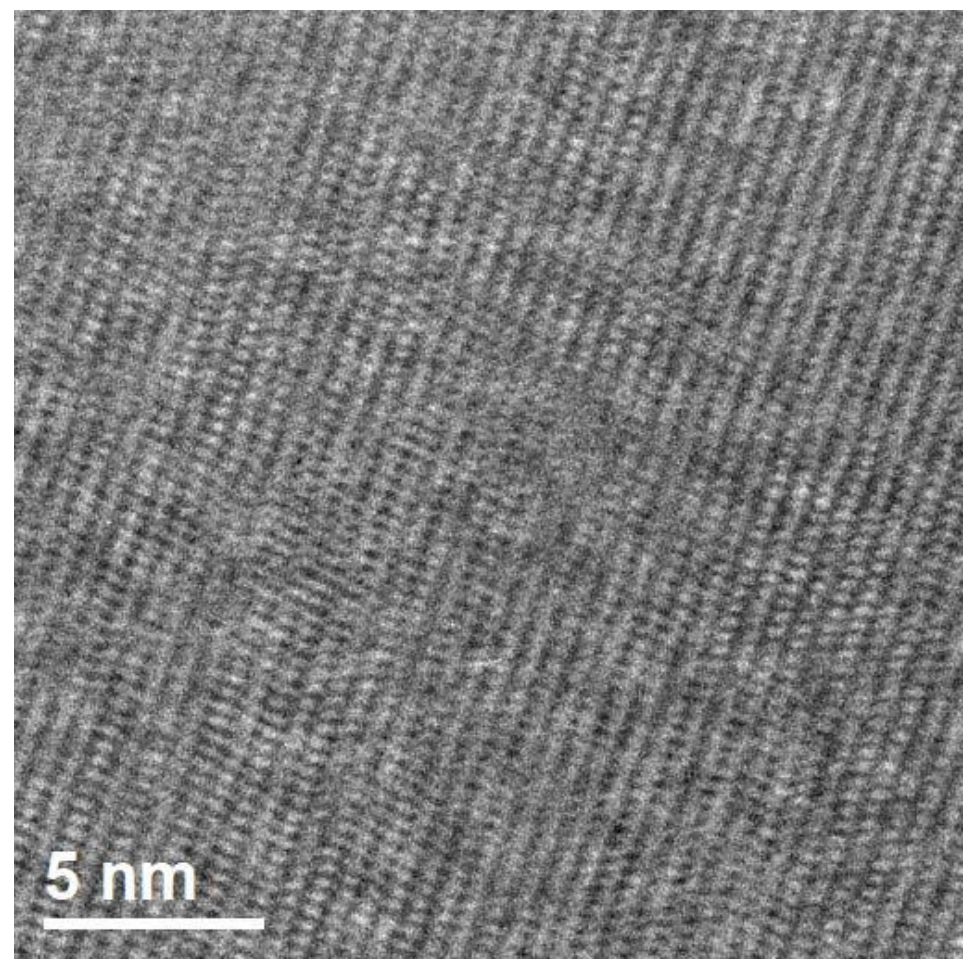

Figure 1. Atomic resolution image of a curved urate crystal.

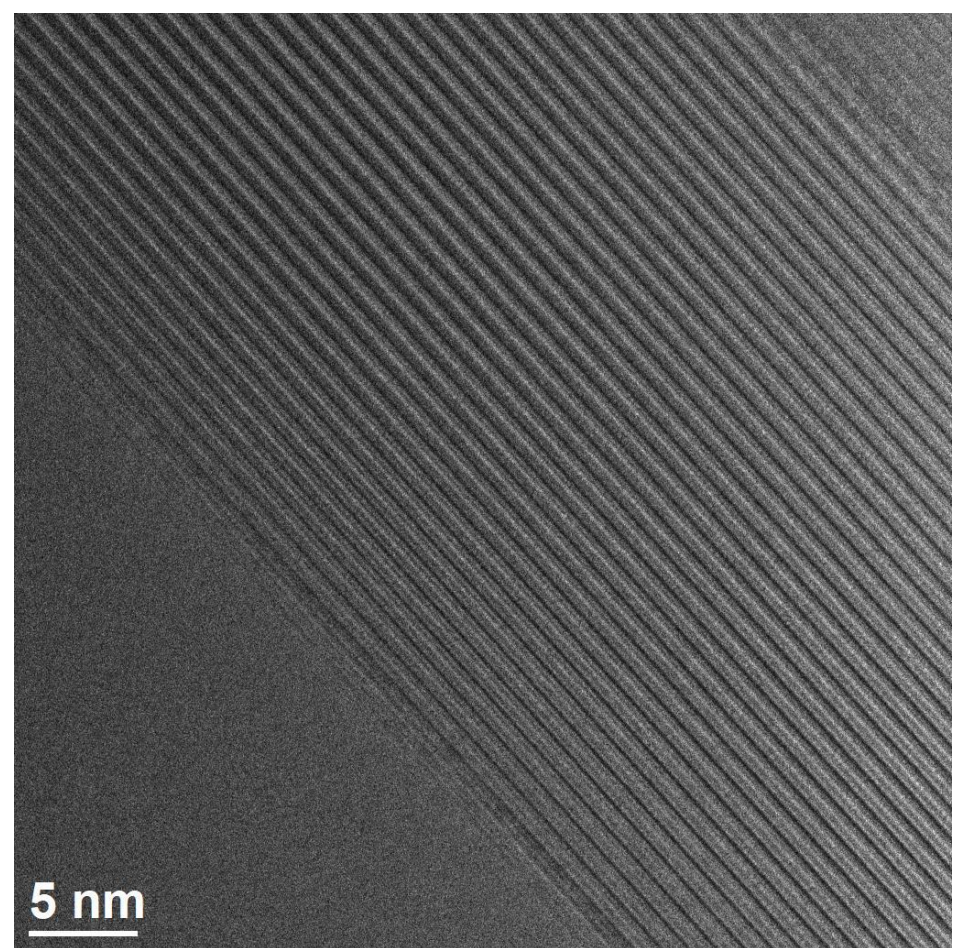

Figure 2. Atomic resolution image of straight urate crystal

\section{References}

1. Work at the molecular Foundry was supported by the Office of Science, Office of Basic Energy Sciences, of the US Department of Energy under Contract No DE-AC02-05CH11231. 